\title{
Resistência do Abacaxizeiro 'Turiaçu' a Meloidogyne arenaria, M. enterolobii, $M$. incognita e M.javanica
}

\author{
Gilson Soares da Silva, Any Izabela Silva Krasucki, Isis Lorena Medeiros Rozário, Raycenne Rosa Leite
}

Universidade Estadual do Maranhão, Departamento de Fitotecnia e Fitossanidade, CEP 65001-970, São Luís, MA.

Autor para correspondência: Gilson Soares da Silva (gilson_soares@uol.com.br)

Data de chegada: 25/11/2014. Aceito para publicação em: 17/06/2015.

$10.1590 / 0100-5405 / 2060$

\section{RESUMO}

Silva, G. S.; Krasucki, A. I.; Rozário, I. L.; Leite. R. R. 2012. Resistência do abacaxizeiro ‘Turiaçu’ a Meloidogyne arenaria, M. enterolobii, M. incognita e M. javanica. Summa Phytopathologica, v.41, n.3, p.227-228, 2015.

Neste trabalho avaliou-se, em condições de casa de vegetação, a reação do abacaxizeiro 'Turiaçu' [Ananas comosus (L.) Merril 'Turiaçu'] a Meloidogyne arenaria, M. enterolobii, M. incognita e M. javanica. Mudas tipo filhote foram plantadas em vasos com capacidade de $3 \mathrm{~L}$ contendo solo autoclavado. Quando as mudas apresentaram raízes desenvolvidas, foram inoculadas com 5000 ovos de cada espécie do nematoide, separadamente.
Foi adotado um delineamento inteiramente casualizado com dez repetições. Cento e cinquenta dias após a inoculação, as plantas foram avaliadas quanto ao fator de reprodução do nematoide ( $\mathrm{FR}=$ população final/população inicial). $\mathrm{O}$ abacaxizeiro 'Turiaçu' mostrou-se altamente resistente às quatro espécies de Meloidogyne, com fatores de reprodução variando entre 0,00 a 0,03 .

Palavras-chave: nematoides das galhas, Ananas comosus,

\section{ABSTRACT}

Silva, G. S., Krasucki, A. I. \& Rozário, I. L.; Leite, R. R. Resistance of pineapple 'Turiaçu' to Meloidogyne arenaria, M. enterolobii, M. incognita and M.javanica. Summa Phytopathologica, v.41, n.3, p.227-228, 2015.

This study evaluated, under greenhouse conditions, the reaction of the pineapple 'Turiaçu' [Ananas comosus (L.) Merril 'Turiaçu'] to Meloidogyne arenaria, M. enterolobii, M. incognita and M. javanica. Slips were planted in 3-L pots containing autoclaved soil. When the seedlings had developed roots, they were inoculated with 5000 eggs of each nematode species separately. A completely randomized design was adopted with ten replicates. One hundred and fifty days after inoculation, the plants were evaluated for the nematode reproduction factor ( $\mathrm{Rf}$ = initial population/final population). The pineapple 'Turiaçu' was highly resistant to the four Meloidogyne species, with reproduction factors ranging from 0.00 to 0.03 .

Additional keywords: root-knot nematodes, Ananas comosus.

Entre os problemas fitossanitários que ocorrem na cultura do abacaxizeiro [ Ananas comosus (L.) Merril], os fitonematoides estão entre os mais importantes, pois causam perdas acentuadas de produção (10). Diversas espécies de fitonematoides já foram assinaladas associadas ao abacaxizeiro, destacando-se os formadores de galhas Meloidogyne incognita (Kofoid \& White) Chitwood e M. javanica (Treub) Chitwood, o nematoide reniforme (Rotylenchulus reniformis Linford \& Oliveira) e o nematoide das lesões radiculares (Pratylenchus brachyurus (Godfrey) Filipjev \& Stekhoven $(4,5,6)$.

O método mais prático de controle desses organismos é a utilização de cultivares resistentes. No Brasil, alguns trabalhos visando identificar fontes de resistência a $M$. incognita, $M$. javanica e $P$. brachyurus já foram realizados $(3,5,9)$. Recentemente, a resistência a $M$. enterolobii foi identificada na cultivar Vitória (9).

O abacaxizeiro 'Turiaçu' ( Ananas comosus 'Turiaçu') é uma cultivar originária do município de Turiaçu, Maranhão. De polpa amarela e elevado índice de doçura, é muito apreciada pela população, alcançando preços superiores em relação às cultivares tradicionais (1). Quanto à sua reação aos nematoides das galhas não há informações a respeito.

Neste trabalho, avaliou-se, em condições de casa de vegetação, o comportamento dessa cultivar a $M$. arenaria, M. enterolobii, $M$. incognita e M.javanica.

Mudas tipo filhote foram plantadas em vasos com capacidade de 3 litros, contendo solo previamente autoclavado. Quando as plantas apresentavam raízes desenvolvidas, quarenta dias após o plantio, foram inoculadas com 5000 ovos de cada espécie de Meloidogyne, separadamente. Como fonte de inóculo foram utilizadas plantas de tomateiro (Solanum lycopersicum L.), goiabeiras 'Paluma' (Psidium guajava L.) e tomateiro infestadas por M. arenaria, M. enterolobii, M. incognita e $M$. javanica, respectivamente, mantidas em casa de vegetação. Para a obtenção do inóculo, os ovos foram extraídos pelo método de Hussey \& Barker (7), modificado por Boneti \& Ferraz (2). As plantas foram inoculadas vertendo-se $10 \mathrm{~mL}$ de uma suspensão de ovos de cada espécie do nematoide, separadamente, na concentração de 500 ovos $/ \mathrm{mL}$, em um sulco feito ao redor do colo das plantas com o auxílio de uma pipeta tipo Oxford. Adotou-se um delineamento experimental inteiramente casualizado com dez repetições. Para 
confirmar a viabilidade do inóculo e para efeito de comparação, tomateiros foram inoculados da mesma forma que os abacaxizeiros e na mesma concentração de inóculo. A avaliação deu-se 150 dias após a inoculação, exceto para os tomateiros que foram avaliados aos 45 dias após a inoculação em razão das condições vegetativas apresentadas, resultado do parasitismo do nematoide. As plantas foram retiradas dos vasos, as raízes lavadas e processadas para a extração dos ovos, calculando-se o fator de reprodução ( $\mathrm{FR}=$ população final/população inicial).

Os resultados obtidos estão apresentados na Tabela 1. As plantas inoculadas não apresentaram sintomas típicos do parasitismo dos nematóides das galhas na parte aérea tais como deficiência mineral, sub-desenvolvimento, dentre outros. O exame dos sistemas radiculares das plantas inoculadas também não evidenciou a presença de galhas nas raízes. Observações semelhantes foram feitas por Lima et al. (8). Inoculando M. enterolobii em quatro cultivares de abacaxizeiro, verificaram que o nematoide não afetou o desenvolvimento vegetativo das plantas.

Tabela 1. Fator de reprodução de Meloidogyne arenaria, M. enterolobii, M. incognita e M. javanica em abacaxizeiro 'Turiaçu', 150 dias após a inoculação.

\begin{tabular}{ccc}
\hline \multirow{2}{*}{ Espécie de Meloidogyne } & \multicolumn{2}{c}{ Fator de Reprodução* } \\
\cline { 2 - 3 } & Abacaxizeiro & Tomateiro \\
\hline Meloidogyne arenaria & 0,03 & 19,3 \\
Meloidogyne enterolobii & 0,00 & 14,8 \\
Meloidogyne incognita & 0,02 & 8,7 \\
Meloidogyne javanica & 0,02 & 7,9 \\
\hline
\end{tabular}

"Fator de reprodução= população final/população inicial.

Para todas as espécies de Meloidogyne avaliadas, o abacaxizeiro 'Turiaçu' mostrou-se altamente resistente, com fatores de reprodução variando de 0,00 a 0,03 . De acordo com os resultados obtidos, a cultivar 'Turiaçu' apresenta-se como uma alternativa econômica bastante atrativa de cultivo para áreas infestadas com nematóides das galhas. Outra característica importante dessa cultivar de abacaxizeiro é a sua resistência à fusariose (Fusarium guttiforme Nirenburg \& O'Donnel, 1998), uma das principais doenças da cultura em todas as regiões produtoras (1).

\section{Agradecimentos}

Os autores agradecem à Dra. Regina M. D. G. Carneiro, da Embrapa Recursos Genéticos e Biotecnologia, pela identificação das espécies de Meloidogyne utilizadas neste trabalho.

\section{REFERÊNCIAS BIBLIOGRÁFICAS}

1. Araújo, J.R.G.; Aguiar Júnior, R.A.; Chaves, A.M.S.; Reis, F.O.; Martins, M.R. Abacaxi 'Turiaçu' : cultivar tradicional nativo do Maranhão. Revista Brasileira de Fruticultura, v.34, n. 4, p. 1270-1276, 2012.

2. Boneti, J.I.S.; Ferraz, S. Modificação do método de Hussey \& Barker para extração de ovos de Meloidogyne exigua de raízes de cafeeiro. Fitopatologia Brasileira, v.6, n.3, p.553, 1981.

3. Costa, D.C.; Cabral, J.R.S.; Calfa, C.H.; Rocha, M.A.C. Seleção de genótipos de abacaxi para resistência a Meloidogyne javanica e Pratylenchus brachyurus. Pesquisa Agropecuária Tropical, v. 29, n.1, p.57-60, 1999.

4. Dias-Arieira, C.R.; Molina, R.O. ; Costa, A.T. Nematoides causadores de doenças em frutíferas. Agro@mbiente, v.2,n.1, p. 46-56, 2008.

5. Dinardo-Miranda, L.L.; Spironello, A.; Martins, A.L.M. Reação de variedades de abacaxizeiro a Pratylenchus brachyurus. Nematologia Brasileira, v.20, n.1, p. 1-7, 1996.

6. Freire, F.C.O. 2003. Nematoides de fruteiras tropicais de interesse agroindustrial. In: Freire, F.C.O. , Cardoso, J.E.; Viana, F. M. P. (Eds.). Doenças de fruteiras tropicais de interesse agroindustrial. Brasília:Embrapa Informação Tecnológica, 2003. p. 493-570.

7. Hussey, R.S.; Barker, K.R. A comparison of methods of collecting inocula for Meloidogyne spp., including a new technique. Plant Disease Reporter, v.57, n. 12, p.1025-1028. 1973.

8. Lima, I. M.; Ventura, J. A.; Caetano, L. C.; Costa, H. ; Oliveira, E. B. Resistência do abacaxizeiro 'Vitória' a Meloidogyne enterolobii. Tropical Plant Pathology, Lavras, v. 36(suplemento): 479. (Resumo). 2011

9. Lima, I.M.; Ventura, J.A.; Caetano, L.C.; Costa, H.; Oliveira, E.B. Desenvolvimento vegetativo de quatro cultivares de abacaxizeiro em área infestada com Meloidogyne enterolobii. Tropical Plant Pathology, Lavras, v. 36 , supl., p. 478. (Resumo), 2011.

10. Sipes, B.S.; Caswell-Chen, E.P.; \& Sarah, J-L; Apt, W.J. Nematodes parasites of pineapple. In: LUC, M.; SIKORA, R. A. \& BRIDGE, J. (Eds.). Plant parasitic nematodes in subtropical and tropical agriculture. $2^{\text {th }}$ ed. London: CABI International, Wallingford UK, 2005. p. 709-731. 